LBNL-51871

UCB-PTH-02/58

UFIFT-HEP-02-36

\title{
An Improved Mean Field Approximation on the Worldsheet for Planar $\phi^{3}$ Theory *
}

\author{
Korkut Bardakci ${ }^{a}$ and Charles B. Thorn ${ }^{b}$ \\ ${ }^{a}$ Department of Physics, University of California at Berkeley \\ ${ }^{a}$ Theoretical Physics Group, Lawrence Berkeley National Laboratory \\ University of California, Berkeley CA 94720 \\ ${ }^{b}$ Institute for Fundamental Theory, Department of Physics \\ University of Florida, Gainesville FL 32611
}

\begin{abstract}
We present an improved version of our earlier work on summing the planar graphs in $\phi^{3}$ field theory. The present treatment is also based on our world sheet formalism and the mean field approximation, but it makes use of no further approximations. We derive a set of equations between the expectation values of the world sheet fields, and we investigate them in certain limits. We show that the equations can give rise to (metastable) string forming solutions.
\end{abstract}

${ }^{*}$ This work was supported in part by the Director, Office of Science, Office of High Energy and Nuclear Physics, of the U.S. Department of Energy under Contract DE-AC0376SF00098, in part by the National Science Foundation Grant 22386-13067-44-X-PHHXM, and in part by the Department of Energy under Grant No. DE-FG01-97ER-41029 


\section{Introduction}

One of the most challenging problems in the study of non-Abelian gauge theories is to discover the dual string description [1]. After many years of relative stagnation, the discovery of the AdS/CFT correspondence $[2,3]$ has led to important progress in the resolution of this problem. This approach relies heavily on ideas from string theory and supersymmetry, and it can deal effectively with only a fairly restricted class of field theories.

Recently, we initiated a program [4], one of the goals of which is to establish the duality between field and string theories. The idea, which goes back to Nielsen, Olesen, Sakita and Virasoro [5], and which was given a systematic foundation via 't Hooft's $1 / N_{c}$ expansion [6], is to construct a world sheet description of the planar graphs of a general field theory. As shown in [6], planar graphs are selected by introducing an internal color degree of freedom and taking $N_{c} \rightarrow \infty$ limit, and the use of a mixed coordinate-momentum space light cone coordinates leads to the world sheet description. Although originally only a $\phi^{3}$ theory was considered for simplicity $[4,6]$, this approach is sufficiently flexible to be able to handle more realistic cases, including nonsupersymmetric [7] and supersymmetric [8] gauge theories.

The original world sheet model suggested by 't Hooft was non-local. In reference [4], it was shown how to reformulate this model as a local world sheet field theory by introducing additional non-dynamical fields. The world sheet structure of a given graph consists of the bulk and a bunch of "solid" lines, which describe the multiple internal boundaries representing the loops of a multi-loop planar diagram. The dynamics is all in the boundaries, and the only function of the fields that live in the bulk is to instantaneously transmit the interaction from one boundary to another. Therefore, the local world sheet theory can be thought of as a topological theory.

The representation of planar graphs by a topological field theory is perhaps elegant, but it has no new physical content. The elimination of the bulk fields would lead back to the original non-local structure of 't Hooft. One gets something new and interesting if the topological bulk fields are some- 
how promoted into genuine dynamical fields. One way for this to happen is through the condensation of the solid lines (the boundaries). In a graph of a given order, the density of the solid lines, by which we mean the percentage of the area on the world sheet occupied by solid lines, is zero. This is because solid lines are lines; they have zero thickness. However, as the order of the graph asymptotes to infinity, solid lines become more and more numerous and dense, and ultimately, one can envisage a limit in which they acquire a finite density on the world sheet. This is what we mean by the condensation of the boundaries. In this limit, the distinction between the bulk and the boundary disappears, the world sheet acquires a uniform texture, and it becomes possible to have a string formulation of the sum of the Feynman graphs in terms of dynamical fields on the world sheet. We will call this mechanism string formation through condensation of boundaries. Whether this really happens depends on the dynamics: One clearly needs an attractive interaction and also an effectively strong coupling, leading to the domination of higher order graphs.

The worldsheet formalism of [4] might also provide a natural setting for understanding confinement in real QCD, where strong coupling is only a feature of infrared dynamics. Then the physical world sheet representing the confining flux tube would not have the uniform texture mentioned above but would include important fluctuations in which regions of the world sheet would not have solid line condensation and would retain the topological character of perturbation theory. These fluctuations would describe the point-like structures implied by asymptotic freedom.

In an earlier paper [9], using $\phi^{3}$ theory as a prototype toy model, we investigated the possibility of boundary condensation leading to string formation. Although this is an unphysical theory, it provides a simple setting for developing the tools needed to attack more interesting and also more complicated theories. The main tool used in [9] was the mean field approximation, or the self consistent field approximation. This approximation has been widely used in both field theory and many body theory, in most cases leading to at least 
qualitatively reasonable answers. For the problem at hand, the easiest way to implement it systematically is to consider the limit of large $D$, where $D$ is the number of transverse dimensions in the light cone picture. We should add that although the large $D$ limit provides a convenient bookkeeping device, it does not truly capture the physics of the problem in most cases. The critical factor is the number of degrees of freedom of the system in question: The mean field approximation is usually successful when applied to a system with a large number of degrees of freedom. Of course, one way to to have a large number of degrees of freedom is to have a large number of space dimensions, but this is rather artificial in most cases. A more physical situation is to have a large number of particles in a many body system, and this is the case in most of the standard applications. In our case, as explained above, we are interested in graphs with a large number of boundaries, and we can roughly identify the number of boundaries with the degrees of freedom. With this identification, the mean field method should be a good tool for investigating problems involving graphs with a large number of boundaries. There is then no need to appeal to the large $D$ limit, except, as a convenient bookkeeping device.

In this article we aim to improve the treatment of [9] in several respects. First, we applied the mean field approximation to a system of Ising spins introduced as a representation of the sum over all arrangements of solid lines. Since the world sheet on which these spins lived remained 2 dimensional even as $D \rightarrow \infty$, it was not clear why that should justify replacing each spin by a mean field. Instead the large $D$ limit is actually that of an $O(D)$ vector model. As is well-known this limit justifies treating the $O(D)$ invariants, the scalar products of the vector fields, classically. In fact the process of replacing the scalars with classical fields eventually leads to a mean field treatment of the Ising spins as well, but, unlike in [9], there are no ambiguities in how to set up the action for the classical mean fields. We incorporate this clarification in the current article. But the most substantial improvement we make here is in the treatment of the Dirichlet boundary conditions. In [9] we introduced 
Gaussian representations of delta functions of the type

$$
\delta(\dot{\mathbf{q}})=\lim _{\beta \rightarrow \infty}\left(\frac{\beta}{2 \pi}\right)^{D / 2} e^{-\beta \dot{\mathbf{q}}^{2}}
$$

to enforce the boundary conditions. Keeping $\beta$ finite amounts to imposing an infrared cutoff on target space. With a sufficiently exact calculation there is nothing wrong with this. But after making an approximation to the cutoff theory, the delicacy of removing the infrared cutoff might well invalidate the approximation. In the current article we shall impose the boundary conditions exactly, thereby eliminating this difficulty. The price will be the need to introduce a more complicated system of mean fields, some of which are nonlocal on the world sheet. The presence of such nonlocal mean fields might even make it easier to incorporate the non-homogeneous worldsheet textures required for a realistic worldsheet description of QCD. We are then able to derive a set of equations for these fields, which relies on no other approximation except for the mean field approximation. We consider these equations (eqs.(46) and (47)) as the main result of this article. Although these equations are non-linear and somewhat complicated, it should be possible to attack them by various approximation schemes and numerical methods. However, in this article, we will be content with studying them in some limiting cases, and we leave a full investigation of this problem to future research.

The paper is organized as follows. In the next section, we present a brief review of the world sheet formalism developed in [4] for summing planar graphs. We also argue that, in the leading order of the mean field approximation, a good deal of simplification takes place: The ghost fields can be dropped, and the world sheet dynamics is described by a free action (eq.(7)), plus constraints on the boundaries given by eq.(8). In section 3 , the boundary constraints are re-expressed in a form suitable for inclusion in the effective action and also for taking the large $D$ limit. This is done by introducing auxiliary scalar fields, which are equal to the scalar products of the vector fields in $D$ dimensions. The important point is that these scalar fields become 
classical in the large $D$ limit. Section 4 is devoted to the construction of the effective action as a function of the scalar fields. This is done by explicitly carrying out the functional integration over the vector fields. The classical equations for the scalar fields resulting from this effective action completely determines the large $D$ dynamics. In section 5 , we investigate these equations in various limits, and we argue that one solution, although not stable, can lead to condensation of the boundaries and string formation. This is a moderately encouraging but preliminary result, although no definite conclusions can be reached until we have the full solution. Section 6 summarizes our conclusions. In the appendix, we show that the contribution of the ghost fields is unimportant in the leading order of the large $D$ limit.

\section{A Brief Review}

We will be working with a massless $\phi^{3}$ matrix field theory in the large $N$ limit, which amounts to a summation of the planar graphs. As 't Hooft has shown [6], Feynman rules are especially simple if a particular mixture of coordinate and momentum light cone variables are used. We will use the following notation: A Minkowski vector $v^{\mu}$ will be written as $\left(v^{+}, v^{-}, \mathbf{v}\right)$, where $v^{ \pm}=\left(v^{0} \pm v^{3}\right) / \sqrt{2}$, and the boldface letters label the components along the transverse directions. The Lorentz invariant product of two vectors $v$ and $w$ is given by $v \cdot w=\mathbf{v} \cdot \mathbf{w}-v^{+} w^{-}-v^{-} w^{+}$. The evolution parameter (time) is $x^{+}$, and the Hamiltonian conjugate to this time is $p^{-}$. A massless onshell particle thus has the energy $p^{-}=\mathbf{p}^{2} / 2 p^{+}$. The number of transverse dimensions $D$ is arbitrary to start with, and eventually, we will consider the large $D$ limit. As explained in [4], this limit is a convenient method of organizing the mean field approximation. Of course, the eventual case of interest is $D=2$.

Let us now briefly review the Feynman rules derived in [6]. A specific graph is represented by a set of parallel solid lines drawn on the world sheet. A propagator corresponds to a strip bounded by two solid lines (Fig.(1)). If $p$ is the momentum carried by the propagator, the strip has width $p^{+}$, and 


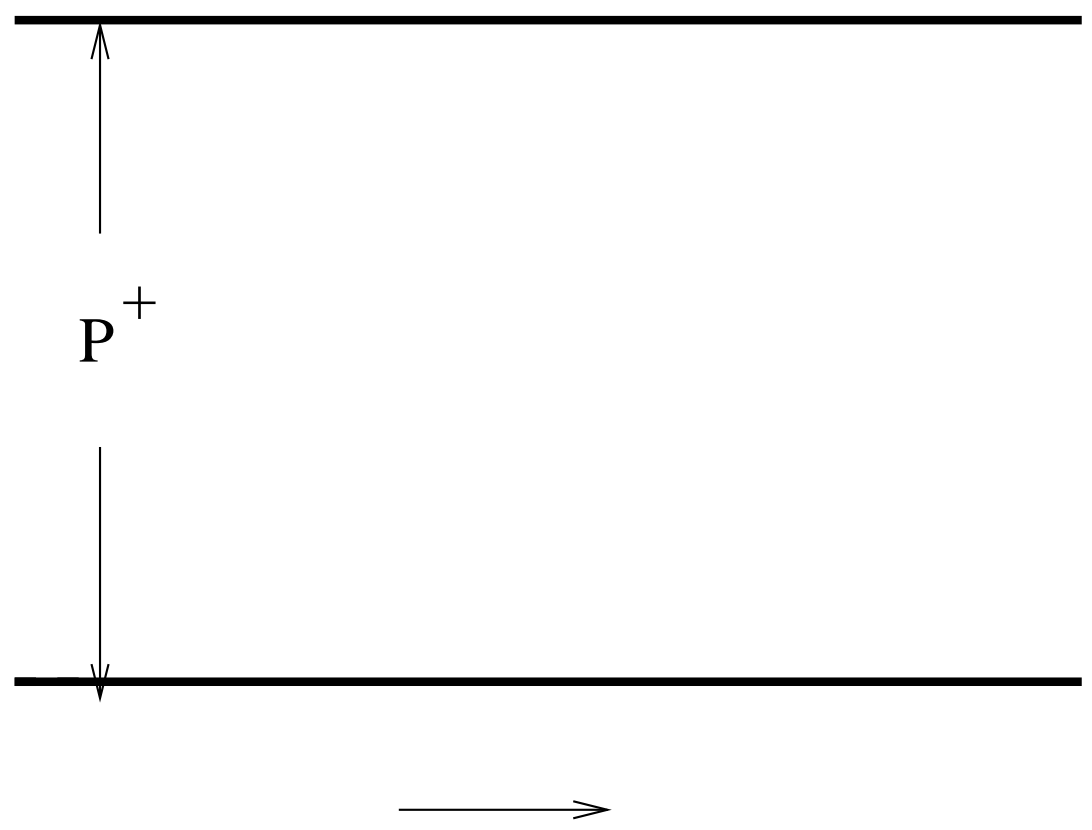

Figure 1: A Propagator

length $\tau=x^{+}$. We associate two transverse momenta $\mathbf{q}_{1}$ and $\mathbf{q}_{2}$ with the two solid lines forming the boundary. The transverse momentum $\mathbf{p}$ of the propagator is their difference:

$$
\mathbf{p}=\mathbf{q}_{1}-\mathbf{q}_{2},
$$

and using a Euclidean world sheet metric, the propagator is given by

$$
\frac{\theta(\tau)}{2 p^{+}} \exp \left(-\tau\left(\mathbf{q}_{1}-\mathbf{q}_{2}\right)^{2} / 2 p^{+}\right),
$$

Now let us consider a more complicated graph, with interaction vertices, pictured in Fig.(2). Interaction takes place at the points where a solid line ends, and a factor of $g$ is associated with each such vertex, where $g$ is the coupling constant. After putting together the propagators and coupling constants associated with the graph, one has to integrate over the positions of 


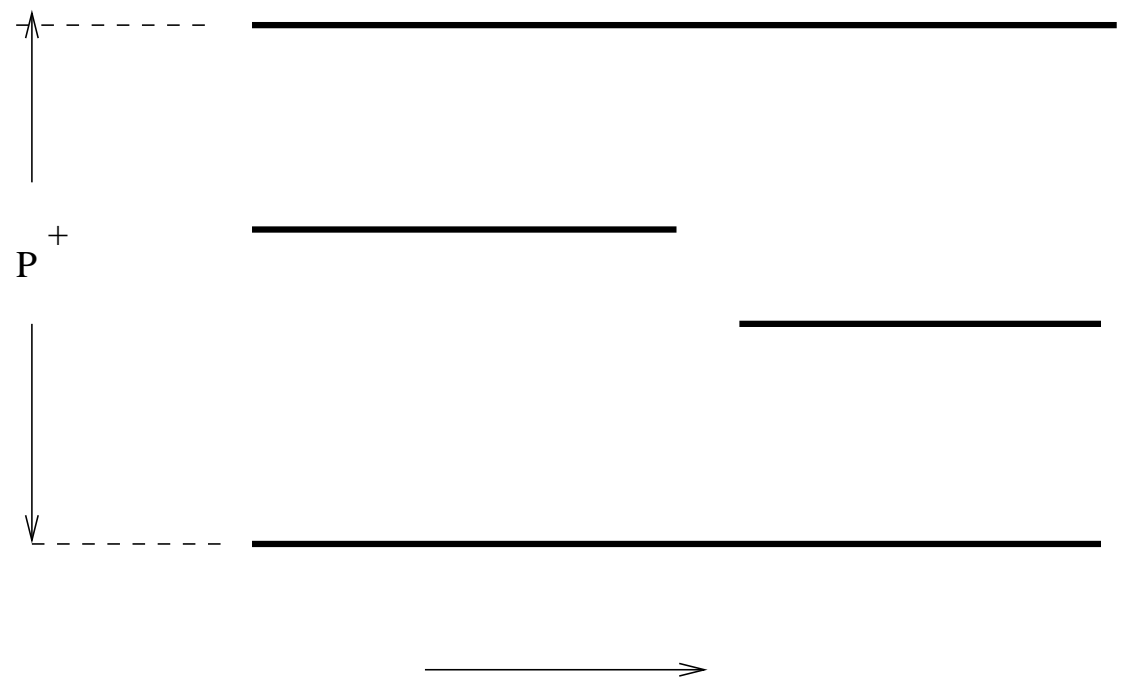

Figure 2: A Typical Graph

the solid lines and the position of the interaction vertices, as well as the momenta carried by solid lines. We note that momentum conservation is automatic in this formalism.

These Feynman rules look quite non-local; however, it was shown in reference [4] that they can be reproduced by a local field theory defined on the world sheet. Later, this result was extended from $\phi^{3}$ to more complicated and more realistic theories $[7,8]$. The world sheet field theory in question can be formulated either on a latticized or continuum world sheet, and here we choose the continuum version. We will eventually need to specify some cutoffs, but we defer that until the need arises. Using the coordinates $\sigma$ in the $p^{+}$direction and $\tau$ itself in the $x^{+}=\tau$ direction, the bosonic fields $\mathbf{q}(\sigma, \tau)$ and the fermionic fields $b(\sigma, \tau)$ and $c(\sigma, \tau)$, called ghosts, are introduced. In contrast to q, which has $D$ components, $b$ and $c$ each have $D / 2$ components. (Here, we are assuming that $D$ is even). The free part of the action on the world sheet is given by

$$
S_{0}=\int_{\tau_{i}}^{\tau_{f}} d \tau \int_{0}^{p^{+}} d \sigma\left(b^{\prime} \cdot c^{\prime}-\frac{1}{2} \mathbf{q}^{\prime 2}\right),
$$


where the prime denotes derivative with respect to $\sigma$. Here $p^{+}$is the + component of the total momentum flowing through the graph. For convenience, we have also restricted the $\tau$ integration; eventually, we will let $\tau_{i} \rightarrow-\infty$ and $\tau_{f} \rightarrow+\infty$. This action is to be supplemented by the following boundary conditions: On solid lines, $\mathbf{q}(\sigma, \tau)$ is constrained to be independent of $\tau$, or, equivalently, the Dirichlet boundary condition

$$
\dot{\mathbf{q}}=0
$$

is imposed, where the dot denotes derivative with respect to $\tau$. Also, if the transverse momentum carried by the whole graph is $\mathbf{p}$, the constraint

$$
\int_{0}^{p^{+}} d \sigma \mathbf{q}^{\prime}=\mathbf{p}
$$

has to be imposed. For simplicity, we will set $\mathbf{p}=0$ in what follows, which means periodic boundary conditions on $\mathbf{q}$ :

$$
\mathbf{q}(\sigma=0)=\mathbf{q}\left(\sigma=p^{+}\right) .
$$

The corresponding boundary conditions on the ghosts are simple:

$$
b=c=0
$$

on the solid lines.

In addition to $S_{0}$, it was shown in [4] that the full action contains interaction terms in the form of the insertion of ghost vertices. These insertions are needed to correctly reproduce the factor $1 / 2 p^{+}$in front of the exponential in the expression (1) for the propagator. If, for example, one integrates the matter and ghost fields of $S_{0}$ with the boundary conditions appropriate for the propagator, without any vertex ghost insertions, the result is

$$
\theta(\tau) \exp \left(-\tau \mathbf{p}^{2} / 2 p^{+}\right),
$$

instead of (1). Local ghost vertex insertions can be arranged to correct this defect. However, we will now argue that this is the correct leading term in the large $D$ expansion. To see this, let us rewrite the propagator in the form

$$
\theta(\tau) \exp \left(-\tau \mathbf{p}^{2} / 2 p^{+}-\ln \left(2 p^{+}\right)\right) .
$$


The first term in the exponent really corresponds to $D$ terms since it is the square of a vector with $D$ components. Consequently, in the large $D$ limit, its contribution is of the order of $D$. In contrast, the contribution of the second term is $D$ independent. Therefore, in the leading order in $D$, which is all we are going to consider in this paper, the factor of $1 / 2 p^{+}$can be dropped. This means that, in the large $D$ limit, we are entitled to work with $S_{0}$ given by eq.(2) and ignore the ghost insertion terms.

We are going to take advantage of one more simplification. Although the fermionic (ghost) part of $S_{0}$ does contribute in the large $D$ limit, it will be shown in the appendix that its contribution harmlessly shifts some of the fields we had already introduced. So, bearing in mind that the fields might need to be interpreted differently, we are going to drop the ghost contributions.

To summarize, the starting point of the present work will be the action

$$
S_{0} \rightarrow-\int_{\tau_{i}}^{\tau_{f}} d \tau \int_{0}^{p^{+}} d \sigma\left(\frac{1}{2} \mathbf{q}^{\prime 2}\right),
$$

plus the Dirichlet boundary conditions

$$
\dot{\mathbf{q}}=0
$$

on solid lines and the periodicity condition given by eq.(4). We would like to stress that the terms we have neglected do contribute in the non-leading orders of the large $D$ expansion.

\section{The Boundary Conditions}

Our goal is to express the sum over all the planar graphs in the form of an effective action. We start with eqs.(7) and (8), which generate the simplified Feynman rules of the large $D$ limit. We would like to rewrite the conditions (8) in a form that can be identified as a contribution to the action, and cast them in a form suitable for taking the large $D$ limit. We note that the boundary conditions decouple in the $\sigma$ direction, and so it is convenient first to discretize the $\sigma$ coordinate into small segments of length $\Delta \sigma=\epsilon_{1}$, and 
to impose the conditions for each discrete value of $\sigma$ separately. (In [9] this cutoff $\epsilon_{1}$ was called $m$ the minimal unit of $p^{+}$in the discretized formulation.) The parameter $\epsilon_{1}$ will play the role of an ultraviolet cutoff on the worldsheet. It should be pointed out that we are making an important change in the way we classify graphs. The customary classification is according to the total number of solid lines, which is the same as counting the powers of the coupling constant. This is analogous to a Fock space description. Instead, we are now going to focus on the distribution of solid lines separately for each value of $\sigma$. This is more analogous to the occupation number description. If one is interested in the condensation of the solid lines, the occupation number description is clearly superior, since counting the number of lines in a condensate is not very useful.

Next, for a fixed value of $\sigma$, we are going to carry out the summation over all possible partitionings of solid lines and over all values of momenta flowing through them. This will result in an integral equation. In particular, we note that the Dirichlet boundary conditions (8) will be taken into account exactly. In this respect, the present treatment is superior to the one given in [9]; in that work, only a weaker version of these boundary conditions was imposed.

In Fig.(3), we have drawn lines located at the discrete values of $\sigma$ and extending in the $\tau$ direction. These lines consist of alternating solid and dotted segments: As before, the solid lines are located where the boundary conditions (8) hold, whereas the dotted lines run through what used to be blank space, where there are no boundary conditions. Now consider a typical line located at some $\sigma$, starting at $\tau_{i}$ and ending at a variable point $\tau$. For simplicity, we assume that this line contains an equal number of solid and dotted line segments, and we call this number $m$. One has then to sum over all possible partitions of this line between alternating solid and dotted segments for a given $m$, and then sum over all $m$. Let $F_{m}\left(\sigma, \tau_{i}, \tau\right)$ be the factor that takes care of (8) on the solid segments. This function satisfies the 


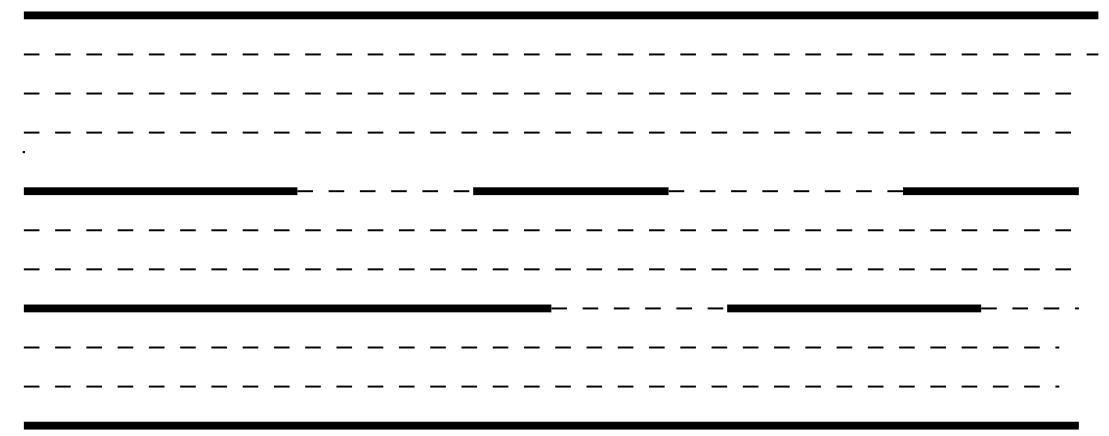

Figure 3: Solid and Dotted Lines

following recursion relation:

$$
F_{m+1}\left(\sigma, \tau_{i}, \tau\right)=g^{2} \int_{\tau_{i}}^{\tau} d \tau_{1} \int_{\tau_{1}}^{\tau} d \tau_{2} K\left(\sigma, \tau_{2}, \tau\right) F_{m}\left(\sigma, \tau_{i}, \tau_{1}\right) .
$$

In going from $F_{m}$ to $F_{m+1}$, we have added a dotted segment extending from $\tau_{1}$ to $\tau_{2}$, and a solid segment extending from $\tau_{2}$ to $\tau$. Since there is no boundary condition on the dotted segment, we associate with it a factor of one. With the solid segment, we associate the factor $K\left(\sigma, \tau_{2}, \tau\right)$, which is introduced to take care of the corresponding boundary condition. The total $F$ is then given by

$$
F\left(\sigma, \tau_{i}, \tau\right)=\sum_{m=0}^{\infty} F_{m}\left(\sigma, \tau_{i}, \tau\right) .
$$

and it satisfies the integral equation

$$
F\left(\sigma, \tau_{i}, \tau\right)=F_{0}\left(\sigma, \tau_{i}, \tau\right)+g^{2} \int_{\tau_{i}}^{\tau} d \tau_{1} \int_{\tau_{1}}^{\tau} d \tau_{2} K\left(\sigma, \tau_{2}, \tau\right) F\left(\sigma, \tau_{i}, \tau_{1}\right) .
$$

So far, we have defined an $F$ that implements (8) for a single line located at $\sigma$. To find the $F$ that implements these boundary conditions for the whole world sheet, we have to multiply the $F$ 's associated with each discrete value of $\sigma$ and let $\tau \rightarrow \tau_{f}$ :

$$
F\left(\tau_{i}, \tau_{f}\right)=\prod_{n} F\left(\sigma_{n}, \tau_{i}, \tau_{f}\right)
$$


Now let us return to the integral equation (11). We will set

$$
F_{0}\left(\sigma, \tau_{i}, \tau\right)=\delta\left(\tau_{i}-\tau\right)
$$

The kernel $K$, which has to implement (8) on the solid line segment extending from $\tau_{2}$ to $\tau$, is given by

$$
K\left(\sigma, \tau_{2}, \tau\right)=\int d \mathbf{k} \int D \mathbf{l} \exp \left(i \int_{\tau_{2}}^{\tau} d \tau^{\prime} \mathbf{l}\left(\sigma, \tau^{\prime}\right) \cdot\left(\mathbf{q}\left(\sigma, \tau^{\prime}\right)-\mathbf{k}\right)\right)
$$

The integration over $\mathbf{l}\left(\sigma, \tau^{\prime}\right)$ sets $\mathbf{q}\left(\sigma, \tau^{\prime}\right)$ equal to a constant $\tau$ independent vector $\mathbf{k}$ in the interval $\left(\tau_{2}, \tau\right)$, and then $\mathbf{k}$ is integrated over. It is clear that this is equivalent to the condition that $\mathbf{q}$ is independent of $\tau^{\prime}$ in this interval.

It will become clear as we proceed that an ultraviolet cutoff in the variable $\mathbf{k}$ is needed to avoid divergences. We therefore modify the expression for $K$ by introducing a suitable cutoff of the form of $\exp \left(-\epsilon k^{2}\right)$ and then do the integration over $\mathbf{k}$ :

$$
\begin{aligned}
& K\left(\sigma, \tau_{1}, \tau_{2}\right) \rightarrow \int d \mathbf{k} \int D \mathbf{l} \exp \left(-\epsilon k^{2}+i \int_{\tau_{1}}^{\tau_{2}} d \tau^{\prime} \mathbf{l}\left(\sigma, \tau^{\prime}\right) \cdot\left(\mathbf{q}\left(\sigma, \tau^{\prime}\right)-\mathbf{k}\right)\right) \\
& =\int D \mathbf{l}(\pi / \epsilon)^{D / 2} \exp \left(i \int_{\tau_{1}}^{\tau_{2}} d \tau^{\prime} \mathbf{l}\left(\sigma, \tau^{\prime}\right) \cdot \mathbf{q}\left(\sigma, \tau^{\prime}\right)\right. \\
& \left.\quad-\frac{1}{4 \epsilon} \int_{\tau_{1}}^{\tau_{2}} d \tau^{\prime} \int_{\tau_{1}}^{\tau_{2}} d \tau^{\prime \prime} \mathbf{l}\left(\sigma, \tau^{\prime}\right) \cdot \mathbf{l}\left(\sigma, \tau^{\prime \prime}\right)\right) .
\end{aligned}
$$

We note that in addition to the worldsheet ultraviolet cutoff $\epsilon_{1}$ introduced earlier, we now have a second ultraviolet cutoff $\epsilon$, acting in the target space. Later, we will find that a third cutoff may be necessary, although it is likely that the third cutoff is expressible in terms of the first two.

It is worth noting that the nonlocal term in eq.(15) is entirely due to the integration over $\mathbf{k}$, the Dirichlet value of $\mathbf{q}$ on the internal boundary which $K$ adds to the worldsheet. But in the worldsheet formalism applied to gauge theories with extended supersymmetry [8], the worldsheet variables $\mathbf{q}(\sigma, \tau)$ were $2+d$ dimensional where $d=2$ for $\mathcal{N}=2$ supersymmetry and $d=6$ for $\mathcal{N}=4$ supersymmetry. The process of dimensional reduction, which leads to the 4 dimensional theory with extended supersymmetry, was 
achieved in [8] by imposing the boundary condition $q^{I}=0$ on all boundaries, external and internal, whenever $I=3,4, \ldots d$. This means that the same components of the vector $\mathbf{k}$ are put to zero and not integrated. In other words the nonlocality is not introduced for the components of $\mathbf{l}$ in the extra dimensions. This worldsheet locality for the dimensions associated with the field theoretic internal degrees of freedom could be associated with the generic appearance of $S_{n}$ factors of the space-time manifold on the strong coupling side of the AdS/CFT correspondence ${ }^{\dagger}$.

We are dealing here with a vector model, and the standard first step in taking the large $N$ (in this case, the large $D$ ) limit, is first to express everything in terms of the scalar products of the vectors. From eq.(15), we see that this goal has been achieved: The kernel $K$ is expressed in terms of the scalar products $\mathbf{q} \cdot \mathbf{l}$ and $\mathbf{l} \cdot \mathbf{l}$. In the next step, the scalar products are replaced by their vacuum expectation values, which are proportional to $\mathrm{D}$, and which we denote by $D \phi_{1}$ and $D \phi_{2}$ respectively:

$$
\begin{aligned}
& \mathbf{l}\left(\sigma, \tau^{\prime}\right) \cdot \mathbf{q}\left(\sigma, \tau^{\prime}\right) \rightarrow\left\langle\mathbf{l}\left(\sigma, \tau^{\prime}\right) \cdot \mathbf{q}\left(\sigma, \tau^{\prime}\right)\right\rangle=D \phi_{1}\left(\sigma, \tau^{\prime}\right), \\
& \mathbf{l}\left(\sigma, \tau^{\prime}\right) \cdot \mathbf{l}\left(\sigma, \tau^{\prime \prime}\right) \rightarrow\left\langle\mathbf{l}\left(\sigma, \tau^{\prime}\right) \cdot \mathbf{l}\left(\sigma, \tau^{\prime \prime}\right)\right\rangle=D \phi_{2}\left(\sigma, \tau^{\prime}, \tau^{\prime \prime}\right) .
\end{aligned}
$$

We would like to make it clear that $\phi_{1}$ and $\phi_{2}$ are fixed classical background fields, not to be integrated over. They are the saddle points that dominate the large $D$ limit, to be determined by minimizing the effective action. The general rule is that vector valued fields are quantum mechanical, and they are to be integrated over. In contrast, scalar valued fields are classical background fields.

The kernel $K$ can now be written solely in terms of $\phi_{1}$ and $\phi_{2}$ :

$$
\begin{aligned}
& K\left(\sigma, \tau_{1}, \tau_{2}\right)= \\
& (\pi / \epsilon)^{D / 2} \exp \left(i D \int_{\tau_{1}}^{\tau_{2}} d \tau^{\prime} \phi_{1}\left(\sigma, \tau^{\prime}\right)-\frac{D}{4 \epsilon} \int_{\tau_{1}}^{\tau_{2}} d \tau^{\prime} \int_{\tau_{1}}^{\tau_{2}} d \tau^{\prime \prime} \phi_{2}\left(\sigma, \tau^{\prime}, \tau^{\prime \prime}\right)\right) .
\end{aligned}
$$

\footnotetext{
${ }^{\dagger}$ We thank J. Maldacena for an illuminating conversation that inspired this suggestion.
} 
Substituting this expression back into eq.(11), we have to solve an integral equation to find $F\left(\tau_{i}, \tau_{f}\right)$. We will reconsider this problem in the next section, after the simplifications following from translation invariance are taken into account. Here we note that once $F$ is known, the total action $S$, which incorporates the boundary condition (8) on solid lines, is given by

$$
\exp (S)=\exp \left(S_{1}\right) F\left(\tau_{i}, \tau_{f}\right),
$$

where,

$$
\begin{aligned}
S_{1} & =\int_{0}^{p^{+}} d \sigma \int d \tau\left(-\frac{1}{2}\left(\mathbf{q}^{\prime}(\sigma, \tau)\right)^{2}+i \lambda_{1}(\sigma, \tau)\left(\mathbf{l}(\sigma, \tau) \cdot \mathbf{q}(\sigma, \tau)-D \phi_{1}(\sigma, \tau)\right)\right) \\
& -i \int_{0}^{p^{+}} d \sigma \int d \tau \int d \tau^{\prime} \lambda_{2}\left(\sigma, \tau, \tau^{\prime}\right)\left(\mathbf{l}(\sigma, \tau) \cdot \mathbf{l}\left(\sigma, \tau^{\prime}\right)-D \phi_{2}\left(\sigma, \tau, \tau^{\prime}\right)\right) .
\end{aligned}
$$

The first term on the right is $S_{0}$ (eq.(7)). In the rest of the terms in $S_{1}$, the Lagrange multipliers $\lambda_{1}$ and $\lambda_{2}$ enforce the definition of $\phi_{1,2}$ given by (16).

\section{The Effective Action}

In this section, we will address the problem of the computation of the effective action. This will involve

a) solving the integral equation (11) for $F$ and

b) doing the functional integrations over the vector-valued fields $\mathbf{q}$ and $\mathbf{l}$, keeping all the scalar valued fields fixed.

We will be able to make only partial progress in solving eq.(11). In contrast, the functional integrations over $\mathbf{q}$ and $\mathbf{l}$ can be carried out in closed form. Once the effective action is derived, the classical fields $\phi_{1,2}$ and $\lambda_{1,2}$ are determined by the equations

$$
\frac{\delta S_{e}}{\delta \phi_{1,2}}=0, \quad \frac{\delta S_{e}}{\delta \lambda_{1,2}}=0 .
$$

The calculations outlined above are greatly simplified by making use of translation invariances in both the $\sigma$ and $\tau$ directions on the world sheet. 
This symmetry means that various background fields are either constants or functions of a single variable:

$$
\begin{aligned}
\lambda_{1}(\sigma, \tau) \rightarrow \lambda_{1}, & \phi_{1}(\sigma, \tau) \rightarrow \phi_{1}, \\
\lambda_{2}\left(\sigma, \tau, \tau^{\prime}\right) \rightarrow \lambda_{2}\left(\tau-\tau^{\prime}\right), & \phi_{2}\left(\sigma, \tau, \tau^{\prime}\right) \rightarrow \phi_{2}\left(\tau-\tau^{\prime}\right),
\end{aligned}
$$

where $\lambda_{1}$ and $\phi_{1}$ are independent of $\sigma$ and $\tau$, and $\lambda_{2}$ and $\phi_{2}$ depend only on the difference $\tau-\tau^{\prime}$. Substituting this in eq.(20), we see that $S_{1}$ will be nonlocal in the $\tau$ coordinate. This makes the problem of finding the minimum of the effective action more difficult; instead of an algebraic equation, we have to solve functional equations in one variable.

Let us now go back to the integral equation (11), taking advantage of the simplification resulting from translation invariance. First, we note that the functions $K$ and $F$ simplify:

$$
K\left(\sigma, \tau_{1}, \tau_{2}\right) \rightarrow K\left(\tau_{2}-\tau_{1}\right), \quad F\left(\sigma, \tau_{1}, \tau_{2}\right) \rightarrow F\left(\tau_{2}-\tau_{1}\right) .
$$

Now, eq.(11) can be formally solved using Fourier transforms. We define

$$
\tilde{K}(\omega)=\frac{1}{2 \pi} \int_{0}^{\infty} d \tau e^{i \omega \tau} K(\tau), \quad \tilde{F}(\omega)=\frac{1}{2 \pi} \int_{0}^{\infty} d \tau e^{i \omega \tau} F(\tau) .
$$

The lower limits of integration start at zero, since both $F$ and $K$ are defined to be zero for negative values of the argument. The solution to eq.(11) is then given by

$$
F(\tau)=\int d \omega e^{-i \omega \tau} \tilde{F}(\omega)=\frac{1}{2 \pi} \int d \omega e^{-i \omega \tau} \frac{\omega}{\omega-2 \pi i g^{2} \tilde{K}(\omega)},
$$

where $\tilde{K}$ can be written in the form

$$
\tilde{K}(\omega)=\frac{1}{2 \pi}\left(\frac{\pi}{\epsilon}\right)^{D / 2} \int_{0}^{\infty} d \tau \exp \left(i \omega \tau+i D \phi_{1} \tau-\frac{D}{4 \epsilon} L(\tau)\right)
$$

and where we have defined

$$
L\left(\tau_{2}-\tau_{1}\right)=\int_{\tau_{1}}^{\tau_{2}} d \tau^{\prime} \int_{\tau_{1}}^{\tau_{2}} d \tau^{\prime \prime} \phi_{2}\left(\tau^{\prime \prime}-\tau^{\prime}\right) .
$$


The contribution of $F$ to the effective action follows from eq.(18):

$$
S=S_{1}+S_{F}, \quad S_{F}=\ln \left(F\left(\tau_{f}-\tau_{i}\right)\right)
$$

Now, let us examine $S_{F}$ in more detail. The time interval $\tau_{f}-\tau_{i}$, which corresponds to $\tau$ in eq.(23), will eventually tend to infinity. It is a standard result in Fourier transform that this limit will be dominated by the lowest lying singularity in the variable $\omega$ of the integrand in eq.(23), which could be the tip of a cut or a pole. Since, being conjugate to time, $\omega$ is energy, a cut corresponds to the continuum and the pole to a bound state. We are particularly interested in bound states, since they can lower the energy of the system and lead to a non-trivial solution to our variational problem. Therefore, we will assume that the lowest lying singularity is a pole ${ }^{\ddagger}$, and we will be looking for the zeroes of the denominator in eq.(24):

$$
\omega-2 \pi i g^{2} \tilde{K}(\omega)=0
$$

If $\omega_{0}$ is a solution to this equation, the corresponding energy is given by

$$
E_{0}=i \omega_{0}
$$

and the corresponding contribution to the action is

$$
S_{F}=-\frac{i}{\epsilon_{1}} p^{+}\left(\tau_{f}-\tau_{i}\right) \omega_{0}
$$

where $\epsilon_{1}$ is the cutoff resulting from the discretization of the interval along the $\sigma$ direction. (See eq.(12)). At this point, we should note that bound states cannot arise in perturbation theory, so our treatment from now on is definitely non-perturbative. There is also the question of the reality of $E_{0}$. In a stable theory, the spectrum should be real and bounded from below; however, since we are dealing with $\phi^{3}$, an intrinsically unstable theory, we

\footnotetext{
${ }^{\ddagger}$ In reference [9], the energy spectrum consisted of only discrete states due to the infrared cutoff introduced in the Gaussian representation of the delta functions imposing Dirichlet boundary conditions.
} 
may end up with complex energies. We will investigate this question in section 5 in the context of a simple model.

For the time being, we cannot proceed any further with the bound state problem without knowing $\phi_{2}$, so we will leave its solution in the implicit form defined by eqs.(24), (25) and (28). Let us now turn to the next problem, that of carrying out the functional integrals over the quantum fields $\mathbf{q}$ and $\mathbf{l}$. Replacing $\lambda_{1}$ and $\lambda_{2}$ in eq.(19) by their translation invariant forms (eq.(21)), this equation can be rewritten as

$$
\begin{aligned}
S_{1} & =S_{1}^{\prime}+S_{1}^{\prime \prime} \\
S_{1}^{\prime} & =i D \int_{0}^{p^{+}} d \sigma\left(-\int d \tau \lambda_{1} \phi_{1}+\int d \tau_{1} \int d \tau_{2} \lambda_{2}\left(\tau_{1}-\tau_{2}\right) \phi_{2}\left(\tau_{1}-\tau_{2}\right)\right) \\
S_{1}^{\prime \prime} & =\int_{0}^{p^{+}} d \sigma \int d \tau\left(-\frac{1}{2} \mathbf{q}^{\prime 2}+i \lambda_{1} \mathbf{l} \cdot \mathbf{q}\right) \\
& -i \int_{0}^{p^{+}} d \sigma \int d \tau_{1} \int d \tau_{2} \lambda_{2}\left(\tau_{2}-\tau_{1}\right) \mathbf{l}\left(\sigma, \tau_{1}\right) \cdot \mathbf{l}\left(\sigma, \tau_{2}\right)
\end{aligned}
$$

It turns out to be more convenient to work in the momentum space. We define

$$
\begin{aligned}
\mathbf{q}(\sigma, \tau) & =\int d p_{0} \sum_{p_{1}} \exp \left(i p_{1} \sigma+i p_{0} \tau\right) \tilde{q}\left(p_{0}, p_{1}\right) \\
\mathbf{l}(\sigma, \tau) & =\int d p_{0} \sum_{p_{1}} \exp \left(i p_{1} \sigma+i p_{0} \tau\right) \tilde{l}\left(p_{0}, p_{1}\right) \\
\lambda_{2}(\tau) & =\int d p_{0} \exp \left(i p_{0} \tau\right) \tilde{\lambda}_{2}\left(p_{0}\right) \\
\phi_{2}(\tau) & =\int d p_{0} \exp \left(-i p_{0} \tau\right) \tilde{\phi}_{2}\left(p_{0}\right)
\end{aligned}
$$

where

$$
p_{1}=2 \pi m / p^{+}, \quad m \in Z,
$$

because of the periodic boundary conditions in the $\sigma$ direction.

Before attempting to do the functional integrals over $\mathbf{q}$ and $\mathbf{l}$, we will examine the classical equations of motion. They will enable us to make a comparison with the standard string action. Written in momentum space, 
the equations of motion are

$$
\begin{aligned}
i \lambda_{1} \tilde{l}-p_{1}^{2} \tilde{q} & =0, \\
\lambda_{1} \tilde{q}-4 \pi \tilde{\lambda}_{2}\left(p_{0}\right) \tilde{l} & =0 .
\end{aligned}
$$

The solution to these equations is

$$
p_{1}^{2}=\frac{i \lambda_{1}^{2}}{4 \pi \tilde{\lambda}_{2}\left(p_{0}\right)} .
$$

The usual string action corresponds to taking

$$
\frac{\lambda_{1}^{2}}{\tilde{\lambda}_{2}\left(p_{0}\right)}=16 \pi i \alpha^{2} p_{0}^{2},
$$

where $\alpha$ is the slope parameter. One can see this as follows: In coordinate space, eq.(35) is equivalent to the action

$$
S_{s}=-\frac{1}{2} \int_{0}^{p^{+}} d \sigma \int d \tau\left(4 \alpha^{2} \dot{\mathbf{q}}^{2}+\mathbf{q}^{\prime 2}\right) .
$$

The Hamiltonian corresponding to this action is the light cone energy $p^{-}$, and the quantized values of $p^{-}$are given by the usual string result

$$
p^{-}=\frac{n \pi}{\alpha p^{+}}
$$

where $n$ is a positive integer. We are really interested in the squares of the masses of the excitations, and these are given by

$$
M^{2}=2 p^{+} p^{-}=\frac{2 n \pi}{\alpha}
$$

since we have set (see eq.(4)) $\mathbf{p}=0$. From this result, we see that the slope parameter is given by $\alpha=\left(2 T_{0}\right)^{-1}=\pi \alpha^{\prime}$, where $\alpha^{\prime}$ is the slope of open string Regge trajectories. A string that is linearly confining at large distances corresponds to a $\tilde{\lambda}_{2}$ that has the dependence given by the eq.(35) for small $p_{0}$, so we are going to investigate whether such a behavior is compatible with our dynamical scheme. 
After this diversion, let us now carry out the integrations over $\tilde{q}$ and $\tilde{l}$ in $S_{1}^{\prime \prime}$. The result is the determinant, which is the product of the eigenvalues $\kappa$ that satisfy the equations

$$
\begin{aligned}
i \lambda_{1} \tilde{l}-p_{1}^{2} \tilde{q} & =\kappa \tilde{q}, \\
i \lambda_{1} \tilde{q}-4 \pi i \tilde{\lambda}_{2}\left(p_{0}\right) \tilde{l} & =\kappa \tilde{l},
\end{aligned}
$$

or

$$
\kappa^{2}+\left(p_{1}^{2}+4 \pi i \tilde{\lambda}_{2}\left(p_{0}\right)\right) \kappa+\lambda_{1}^{2}+4 \pi i p_{1}^{2} \tilde{\lambda}_{2}\left(p_{0}\right)=0
$$

The eigenvalues depend on both $p_{0} \equiv p$, which is continuous, and on

$$
p_{1}=\frac{2 \pi m}{p^{+}}
$$

with $m$ an integer. After the integration over $\tilde{q}$ and $\tilde{l}$, we end up with the factor

$$
(\text { det })^{-D / 2}=\prod_{m} \prod_{p}\left(4 \pi i\left(\frac{2 \pi m}{p^{+}}\right)^{2} \tilde{\lambda}_{2}(p)+\lambda_{1}^{2}\right)^{-D / 2}
$$

The corresponding $\operatorname{Tr} \ln$ is given by

$$
-\frac{D}{2} \operatorname{Tr} \ln =-\frac{D\left(\tau_{f}-\tau_{i}\right)}{4 \pi}\left(\int d p\left(\sum_{m} \ln \left(\left(\frac{2 \pi m}{p^{+}}\right)^{2}-\frac{i \lambda_{1}^{2}}{4 \pi \tilde{\lambda}_{2}(p)}\right)+\frac{p^{+}}{\epsilon_{1}} \ln \left(\tilde{\lambda}_{2}(p)\right)\right)\right) .
$$

In writing the right hand side of this equation, we have factored $\tilde{\lambda}_{2}$ from the first term on the right, and we incorporated it into the second term in the form of $\ln \left(\tilde{\lambda}_{2}\right)$. The coefficient of this term involves a sum over $\mathrm{m}$ and appears to be divergent. However, remembering that the $\sigma$ variable was discretized into segments of length $\epsilon_{1}$, this sum is easily seen to be equal to $p^{+} / \epsilon_{1}$.

We now collect various contributions to the effective action:

$$
S_{e}=S_{1}^{\prime}+S_{F}-\frac{D}{2} T r \ln .
$$

The terms that appear in this formula are given in eqs.(31), (30) and (38). It is now easy to write down various classical equations of motion that follow from this action. The equation obtained by varying with respect to $\tilde{\lambda}_{2}$ is

$$
2 \pi i D p^{+}\left(\tau_{f}-\tau_{i}\right) \tilde{\phi}_{2}(p)-\frac{D}{2} \frac{\delta(\operatorname{Tr} \ln )}{\delta\left(\tilde{\lambda}_{2}(p)\right)}=0
$$


or

$$
\tilde{\phi}_{2}(p)=\frac{\lambda_{1}^{2}}{(4 \pi)^{3} \tilde{\lambda}_{2}^{2}(p) s^{1 / 2}(p)} \operatorname{coth}\left(\frac{p^{+}}{2} s^{1 / 2}(p)\right)-\frac{i}{8 \pi^{2} \epsilon_{1} \tilde{\lambda}_{2}(p)},
$$

where, for convenience, we have defined

$$
s(p)=-\frac{i \lambda_{1}^{2}}{4 \pi \tilde{\lambda}_{2}(p)} .
$$

Eq.(41) is the first fundamental equation of our dynamical scheme; it establishes a relation between $\phi_{2}$ and $\lambda_{2}$. A second relation follows from varying the action with respect to $\phi_{2}$ :

$$
\frac{\delta\left(S_{e}\right)}{\delta\left(\phi_{2}\right)}=0
$$

In this case, it turns out to be more convenient to stay in position space and use $L$ instead of $\phi_{2}$ as the independent function. The connection between them is

$$
2 \phi_{2}(\tau)=L^{\prime \prime}(\tau)
$$

where we have assumed that $\tilde{\phi}_{2}(p)$ is an even function of $p$. This assumption, although not crucial, simplifies matters. It is consistent with all our equations and with the approximate solutions given in Section 5 .

Taking into account the contribution of $S_{1}^{\prime}$ (eq.(31)) and of $S_{F}$ (eq.(30)), we have

$$
D \lambda_{2}^{\prime \prime}(\tau)=\frac{2}{\epsilon_{1}} \frac{\delta\left(\omega_{0}\right)}{\delta(L(\tau))}
$$

We have now to compute the functional derivative of $\omega_{0}$ with respect to $L$ from eq.(28). Differentiating this equation gives

$$
\frac{\delta\left(\omega_{0}\right)}{\delta(L(\tau))}=2 \pi i g^{2} \frac{\delta\left(\tilde{K}\left(\omega_{0}\right)\right)}{\delta(L(\tau))}\left(1-2 \pi i g^{2} \tilde{K}^{\prime}\left(\omega_{0}\right)\right)^{-1}
$$

and from eq.(25), we find

$$
\frac{\delta\left(\tilde{K}\left(\omega_{0}\right)\right)}{\delta(L(\tau))}=-\frac{D}{8 \pi \epsilon}\left(\frac{\pi}{\epsilon}\right)^{D / 2} \exp \left(i \omega_{0} \tau+i D \phi_{1} \tau-\frac{D L(\tau)}{4 \epsilon}\right) .
$$


Eqs(42),(43) and (44) put together give us the second relation between $\phi_{2}$, or equivalently $L$, and $\lambda_{2}$ :

$$
\lambda_{2}^{\prime \prime}(\tau)=-\frac{i g_{0}^{2} \exp \left(i \omega_{0} \tau+i D \phi_{1} \tau-\frac{D L(\tau)}{4 \epsilon}\right)}{2 \epsilon \epsilon_{1}\left(1+g_{0}^{2} \int_{0}^{\infty} d \tau \tau \exp \left(i \omega_{0} \tau+i D \phi_{1} \tau-\frac{D L(\tau)}{4 \epsilon}\right)\right)},
$$

where the dimensionless coupling constant $g_{0}$ is defined by

$$
g_{0}^{2}=g^{2}\left(\frac{\pi}{\epsilon}\right)^{D / 2}
$$

To the above equations, one has to add the equations resulting from varying the action with respect to $\lambda_{1}$ and $\phi_{1}$ :

$$
\begin{aligned}
\lambda_{1} \phi_{1} & =4 \pi \int d p\left(\tilde{\lambda}_{2}(p) \tilde{\phi}_{2}(p)+\frac{i}{8 \pi^{2} \epsilon_{1}}\right) \\
\lambda_{1} & =2 i \epsilon \int_{0}^{\infty} d \tau \tau \lambda_{2}^{\prime \prime}(\tau) .
\end{aligned}
$$

We now have a complete set of equations needed to solve for the classical fields $\phi_{1,2}$ and $\lambda_{1,2}$. We would like to stress that, apart from the large $D$ limit, which is the basis of the mean field method, so far everything is exact. Unfortunately, to make progress, we need to know $\omega_{0}$, which is only given implicitly through eq.(28). Ultimately, it should not be too difficult to find approximate solutions by numerical methods. We will, however, leave this problem to future research. Instead, in the next section, we will try to extract as much information as we can from these equations by analytic methods. Such information should be useful for the eventual numerical work.

\section{Consequences of the Dynamical Equations}

In the last section, we have derived a complete set of equations for the dynamical variables $\lambda_{1,2}$ and $\phi_{1,2}$. This section will be devoted to the investigation of the consequences of these equations. First, for the convenience of the reader, we collect the the set of equations to be investigated; namely, eqs.(28),(41) and (45):

$$
0=\omega_{0}-i g_{0}^{2} \int_{0}^{\infty} d \tau \exp \left(i \omega_{0} \tau+i D \phi_{1} \tau-\frac{D}{4 \epsilon} L(\tau)\right)
$$




$$
\begin{aligned}
\tilde{\phi}_{2}(p) & =\frac{\lambda_{1}^{2}}{(4 \pi)^{3} \tilde{\lambda}_{2}^{2}(p) s^{1 / 2}(p)} \operatorname{coth}\left(\frac{p^{+}}{2} s^{1 / 2}(p)\right)-\frac{i}{8 \pi^{2} \epsilon_{1} \tilde{\lambda}_{2}(p)} \\
\lambda_{2}^{\prime \prime}(\tau) & =-\frac{i g_{0}^{2} \exp \left(i \omega_{0} \tau+i D \phi_{1} \tau-\frac{D L(\tau)}{4 \epsilon}\right)}{2 \epsilon \epsilon_{1}\left(1+g_{0}^{2} \int_{0}^{\infty} d \tau \tau \exp \left(i \omega_{0} \tau+i D \phi_{1} \tau-\frac{D L(\tau)}{4 \epsilon}\right)\right)},
\end{aligned}
$$

where

$$
L(\tau)=\int \frac{d p}{p^{2}}\left(2-e^{i p \tau}-e^{-i p \tau}\right) \tilde{\phi}_{2}(p),
$$

which follows from eq.(26).

We will now try to find an approximate solution to eqs.(47), and the approximation will be based on a pole dominance model. This is a crude model, which is at best valid only for large $\tau$, or equivalently, for small $p$. We remind the reader that this region is of interest in probing string formation (see the section following eq.(35)), so any information gleaned is of value. Our starting point is the ansatz

$$
\tilde{\phi}_{2} \rightarrow \beta p^{2}
$$

as $p \rightarrow 0$, where $\beta$ is a constant. The basic idea is to cycle this limiting behavior through the dynamical equations to check its consistency. We first need to restate it in the position $(\tau)$ space. Consider the $\tau \rightarrow \infty$ limit of $L(\tau)$ from eq.(48). In the terms involving the exponentials, this limit can be deduced from the well known Fourier relation: Large $\tau$ is dominated by small $p$. Since the integrand is non-singular at $p=0$ by virtue of the ansatz on $\tilde{\phi}_{2}$, the exponential factors oscillate to zero for $\tau \rightarrow \infty$, with the result that

$$
L(\tau) \rightarrow L_{0}
$$

where $L_{0}$ is a constant. This constant is given by

$$
L_{0}=\int \frac{d p}{p^{2}} \tilde{\phi}_{2}(p)
$$

If $L(\tau)$ goes to a constant as $\tau \rightarrow \infty$, the integral for $\tilde{K}(\omega)$ (eq. (25)) develops a pole in the variable $\omega$. We will now make the assumption that this 
pole is a good approximation for $\tilde{K}$ for small values of $\omega$ (pole dominance). We therefore have

$$
\tilde{K}(\omega) \approx \frac{1}{2 \pi}\left(\frac{\pi}{\epsilon}\right)^{D / 2} \exp \left(-\frac{D L_{0}}{4 \epsilon}\right) \frac{i}{\omega+D \phi_{1}} .
$$

Eq.(28) now becomes

$$
\omega_{0}^{2}+D \phi_{1} \omega_{0}+\bar{g}^{2}=0
$$

where we have defined

$$
\bar{g}^{2}=g_{0}^{2} \exp \left(-\frac{D L_{0}}{4 \epsilon}\right)
$$

The two solutions to this equation are

$$
\omega_{0}^{ \pm}=-\frac{1}{2} D \phi_{1} \pm \frac{i}{2}\left(4 \bar{g}^{2}-D^{2} \phi_{1}^{2}\right)^{1 / 2}
$$

We will investigate both solutions.

Next, we evaluate $\lambda_{2}$ by replacing $L(\tau)$ by $L_{0}$ on the right hand side of eq.(45). As explained above, this should be a good approximation in the large $\tau$, small $p$ regime. Transforming into the momentum space, we have

$$
\begin{aligned}
\tilde{\lambda}_{2}(p) & =-\frac{\bar{g}^{2}}{4 \pi \epsilon \epsilon_{1}} \frac{\left(\omega_{0}+D \phi_{1}\right)^{2}}{\left(\left(\omega_{0}+D \phi_{1}\right)^{2}-\bar{g}^{2}\right)\left(\omega_{0}+D \phi_{1}-p\right)} \frac{1}{p^{2}} \\
& \stackrel{p \rightarrow 0}{\rightarrow} \pm \frac{i \bar{g}^{2}}{4 \pi \epsilon \epsilon_{1} p^{2}}\left(4 \bar{g}^{2}-\left(D \phi_{1}\right)^{2}\right)^{-1 / 2}
\end{aligned}
$$

We note that the $1 / p^{2}$ dependence of $\tilde{\lambda}_{2}$ for small $p$ is exactly what is needed for string formation (see eq.(35)). We can now substitute this result in eq.(41) to determine the small p behavior of $\tilde{\phi}_{2}$, to see whether it is consistent with our initial ansatz (49). In the limit $p \rightarrow 0$, we indeed find that

$$
\begin{aligned}
\tilde{\phi}_{2}(p) & \rightarrow \frac{i}{8 \pi^{2} \tilde{\lambda}_{2}(p)}\left(\frac{1}{p^{+}}-\frac{1}{\epsilon_{1}}\right) \\
& \rightarrow \operatorname{const} \cdot p^{2}
\end{aligned}
$$

establishing consistency. 
It is also important to establish the reality properties of various variables within the context of our approximate solution. We will initially assume that $\phi_{1}$ is pure imaginary and $\bar{g}^{2}$ is real and positive. It then follows from eq.(56) that, at least for small $p, \tilde{\lambda}_{2}$ is pure imaginary. In the same small $p$ limit, eq.(57) tells us that $\tilde{\phi}_{2}$ is real, and therefore, from eq.(51), so is $L_{0}$. We are now able to verify one of our initial assumptions: A real $L_{0}$ means that $\bar{g}^{2}$ is real and positive (eq.(54)). It then follows from eq.(55) that $\omega_{0}^{ \pm}$ are pure imaginary, and the corresponding energies $E_{0}^{ \pm}$(eq.(29)) are real, as they should be. Next, we will investigate $\lambda_{1}$. It can be calculated from the second eq.(46), where $\lambda_{2}^{\prime \prime}$ is given by eq.(45), with $L(\tau)$ replaced by $L_{0}$. The result is

$$
\lambda_{1}=\frac{\bar{g}^{2}}{\epsilon_{1}} \frac{1}{\bar{g}^{2}-\left(\omega_{0}+D \phi_{1}\right)^{2}},
$$

which shows that $\lambda_{1}$ is real. Finally, we go back to the first equation (46): Since $\lambda_{1}$ and $\tilde{\phi}_{2}$ are real, and $\tilde{\lambda}_{2}$ is pure imaginary, it follows that $\phi_{1}$ is pure imaginary, verifying the remaining initial assumption.

From the above analysis, we have seen that $\tilde{\lambda}_{2}(p)$ goes like $p^{2}$ for small $p$, which is the necessary condition for string formation. However, in addition, the square of the slope parameter $\alpha$ (eq.(35)) must be real and positive. Using eq.(56), this parameter is given by

$$
\alpha^{2}=\frac{\lambda_{1}^{2}}{16 \pi i p^{2} \tilde{\lambda}_{2}(p)}=\mp \frac{\epsilon \epsilon_{1} \lambda_{1}^{2}}{4 \bar{g}^{2}}\left(4 \bar{g}^{2}-D^{2} \phi_{1}^{2}\right)^{1 / 2},
$$

and remembering that $\lambda_{1}$ is real and $\phi_{1}$ is pure imaginary, $\alpha^{2}$ is positive for the lower sign. This means that in eq.(55) for $\omega_{0}$, we must also choose the lower sign. This is then the string forming solution, and from the same equation, we see that the corresponding energy $E_{0}^{-}$is positive. However, the other solution, corresponding to the upper sign, has lower energy, since $E_{0}^{+}$is negative, so that it will actually dominate the worldsheet path integral, and not describe a string. The state that looks like string is at best meta-stable and more likely completely unstable. This is, of course, not unexpected, since $\phi^{3}$ is an inherently unstable theory. We expect a more realistic theory, 
such as a non-Abelian gauge theory, to retain the string forming solution but to be free of the instability. As explained in the introduction, our goal in studying $\phi^{3}$ is to develop the tools needed to attack more realistic but also more complicated theories in a simpler setting. We find it encouraging that, despite the instability, we find a signal for string formation.

The results discussed above were based on the pole dominance approximation. As we have argued earlier, this is probably a reasonable approximation for small $p$. In fact, the $1 / p^{2}$ dependence of $\tilde{\lambda}_{2}$ for small $p$, which leads to string formation, appears to be generic. This is easily seen from eq.(45): In momentum space, the double derivative on $\lambda_{2}$ turns into a factor of $p^{2}$, and unless the right hand side of this equation vanishes accidentally at $p=0$, $\tilde{\lambda}_{2}$ must have a $1 / p^{2}$ dependence. On the other hand, we cannot expect the pole dominance model to be valid for large $p$. In fact, we show below that the large $p$ regime is quite different from the small $p$ regime.

We will try to determine the large $p$ behavior of the fields through self consistency. We start with the ansatz

$$
\tilde{\phi}_{2}(p) \rightarrow b|p|,
$$

for $p \rightarrow \infty$, where $b$ is a constant, or, equivalently

$$
\phi_{2}(\tau) \rightarrow-\frac{2 b}{\tau^{2}}
$$

as $\tau \rightarrow 0$. It then follows from

$$
2 \phi_{2}(\tau)=L^{\prime \prime}(\tau)
$$

that

$$
L(\tau) \rightarrow 4 b \ln (\tau),
$$

in the same limit. Eq.(45) then gives

$$
\lambda_{2}(\tau) \rightarrow \text { const } \cdot \tau^{(2-b D / \epsilon)}
$$

again, as $\tau \rightarrow 0$. The motivation for the original ansatz was in fact to obtain a power behavior for $\lambda_{2}$, which translates into

$$
\tilde{\lambda}_{2}(p) \rightarrow \text { const } \cdot|p|^{b D / \epsilon-3}
$$


for large $p$ in momentum space. Now, from eq.(41), we find that

$$
\tilde{\phi}_{2}(p) \rightarrow \text { const } \cdot \tilde{\lambda}(p)^{-3 / 2} \rightarrow \text { const } \cdot|p|^{9 / 2-3 b D / 2 \epsilon},
$$

and comparing this with the initial ansatz, we see that it is consistent if

$$
\frac{b D}{\epsilon}=\frac{7}{3} \text {. }
$$

Admittedly, this is a crude analysis, but if we accept it, we have asymptotic limit

$$
\tilde{\lambda}_{2}(p) \rightarrow \text { const } \cdot|p|^{-2 / 3}, \quad \tilde{\phi}_{2}(p) \rightarrow \text { const } \cdot|p|,
$$

for $p \rightarrow \infty$. This is quite different from the small $p$ behavior

$$
\tilde{\lambda}_{2}(p) \rightarrow \text { const } \cdot 1 / p^{2}, \quad \tilde{\phi}_{2}(p) \rightarrow \text { const } \cdot p^{2} .
$$

We end this section with a short discussion of the need for a third cutoff. The asymptotic behavior derived above shows that the integral for $L$ (eq.(48), as well as the integral for $\lambda_{1} \phi_{1}$ (eq.(46)), are divergent at large $p$. The integrals have to be regulated, say, by a factor of the form

$$
\exp \left(-\epsilon_{2} p^{2}\right)
$$

where $\epsilon_{2}$ is a third cutoff, in addition to $\epsilon$ and $\epsilon_{1}$. This new cutoff is probably not independent of $\epsilon$. The argument goes as follows: Instead of an exponential cutoff, let us latticize the $\tau$ direction, with a lattice spacing $\Delta \tau$. From (6), we see that one lattice step $\Delta \tau$ corresponds to a factor of

$$
\exp \left(-\Delta \tau \mathbf{p}^{2} / 2 p^{+}\right) .
$$

Comparing this with the cutoff $\exp \left(-\epsilon k^{2}\right)$ (eq.(15)), we can identify

$$
\Delta \tau \approx \epsilon p^{+} .
$$

On the other hand, there is a rough relation between $\Delta \tau$ and $\epsilon_{2}$ :

$$
\epsilon_{2} \approx(\Delta \tau)^{2}
$$


and therefore, it follows that

$$
\epsilon_{2} \approx\left(\epsilon p^{+}\right)^{2}
$$

In view of this connection between $\epsilon$ and $\epsilon_{2}$, it is tempting to conjecture that one of them is redundant. In fact, in eqs.(46) and (47), $\epsilon$ and $\epsilon_{1}$ do not regulate any divergent integrals; they merely appear as parameters. One can easily eliminate the singular dependence on these parameters by the following scaling of the fields:

$$
\begin{aligned}
& \phi_{2} \rightarrow \epsilon \phi_{2}, \quad \lambda_{2} \rightarrow \frac{\lambda_{2}}{\epsilon \epsilon_{1}}, \\
& \lambda_{1} \rightarrow \frac{\lambda_{1}}{\epsilon_{1}}, \quad \phi_{1} \rightarrow \phi_{1}, \quad \omega_{0} \rightarrow \omega_{0} .
\end{aligned}
$$

After this scaling, as far as the equations are concerned, one can let

$$
\epsilon \rightarrow 0, \quad \epsilon_{1} \rightarrow 0
$$

while keeping the dimensionless coupling constant $g_{0}$ fixed. Let us see how some physical quantities behave under this scaling. As indicated above, $\omega_{0}$ and therefore $E_{0}$ are independent of $\epsilon$ and $\epsilon_{1}$, whereas the slope parameter $\alpha^{2}$ scales as $\epsilon / \epsilon_{1}$ (eq.(35)). Keeping this ratio finite as each cutoff parameter goes to zero would avoid any singular behavior. So it appears that at least in the leading order of the mean field approximation, both $\epsilon$ and $\epsilon_{1}$ are redundant. In contrast, as pointed out earlier, the cutoff $\epsilon_{2}$ cannot be dispensed with; it is really needed to regulate integrals over $p$ in equations (46) and (48). Clearly, the problems involving cutoff dependence and renormalization must await a better understanding of the solution to eqs.(47).

\section{Conclusions}

The main result of this article is the derivation of a set of equations for the sum of all planar graphs the $\phi^{3}$ field theory, subject to the mean field or large $D$ approximation. Apart from this single approximation, the treatment is exact. This is in contrast to our earlier paper [9], where the same problem 
was considered, but also further and somewhat questionable approximations were made. The present treatment is therefore a definite improvement over the one presented in [9].

The equations derived in this article are somewhat involved but they appear treatable by numerical methods. As a preliminary step, we have studied them in the large and small momentum regimes. The latter is especially important since it is relevant to string formation. We find that a string forming solution is consistent with our equations, although this solution is unstable. Given that we are dealing with an unstable theory to start with, we still find this result encouraging.

Much still remains to be done. A thorough numerical analysis of the equations derived here should be carried out. Since we do not necessarily expect to find interesting results for the $\phi^{3}$ model, the methods developed in this article should be applied to more physical theories, whose world sheet formulations are already available $[7,8]$. It is also important to go to next to leading order in the large $D$ expansion, both as a check on the leading order, and also to investigate questions such as Lorentz invariance.

\section{Acknowledgments}

We would like to thank Jeff Greensite for valuable discussions. This work was supported in part by the Director, Office of Science, Office of High Energy and Nuclear Physics, of the U.S. Department of Energy under Contract DEAC03-76SF00098, in part by the National Science Foundation Grant 2238613067-44-X-PHHXM, and in part by the Department of Energy under Grant No. DE-FG01-97ER-41029 


\section{Appendix. Contribution of the Ghost Sector}

In this appendix, we will discuss the changes resulting from taking into account the ghost fields $b$ and $c$. First of all, an additional factor has to be added to $K$ given by (14):

$$
K \rightarrow K \times K_{g}
$$

where,

$K_{g}\left(\sigma, \tau_{2}, \tau\right)=\int D \bar{b} \int D \bar{c} \exp \left(i \int_{\tau_{2}}^{\tau} d \tau^{\prime}\left(\bar{b}\left(\sigma, \tau^{\prime}\right) \cdot b\left(\sigma, \tau^{\prime}\right)+\bar{c}\left(\sigma, \tau^{\prime}\right) \cdot c\left(\sigma, \tau^{\prime}\right)\right)\right)$

Here, $\bar{b}$ and $\bar{c}$ are lagrange multipliers that enforce the boundary conditions (5) on solid lines. Moreover, there is a ghost contribution to the ac$\operatorname{tion}($ eq. $(28))$ :

$$
S \rightarrow S+S_{g}
$$

where,

$$
S_{g}=\int_{0}^{p^{+}} \int d \tau\left(b^{\prime} \cdot c^{\prime}+\kappa_{1}\left(\bar{b} \cdot b-D \eta_{1}\right)+\kappa_{2}\left(\bar{c} \cdot c-D \eta_{2}\right)\right) .
$$

In this expression, the first term comes from eq.(2), and in the rest of the terms, the lagrange multipliers $\kappa_{1,2}$ set the expectation value of $\bar{b} \cdot b$ and $\bar{c} \cdot c$ equal to $\eta_{1,2}$. As before, this is in preparation for taking the large $D$ limit: Vector valued fields $\bar{b}$ and $\bar{c}$ are traded for scalar valued fields $\kappa_{1,2}$ and $\eta_{1,2}$.

Next, as in eq.(22), we use translation invariance to replace the fields $\kappa_{1,2}$ and $\eta_{1,2}$ by constants independent of $\sigma$ and $\tau$. As a result, we can set

$$
K_{g} \rightarrow \exp \left(i D\left(\eta_{1}+\eta_{2}\right)\left(\tau-\tau_{2}\right)\right)
$$

and eq.(18) gets replaced by

$$
K \rightarrow(\pi / \epsilon)^{D / 2} \exp \left(i D\left(\tau_{2}-\tau_{1}\right)\left(\phi_{1}+\eta_{1}+\eta_{2}\right)-\frac{D}{4 \epsilon} L\left(\tau_{2}-\tau_{1}\right)\right) .
$$

We see that as far as $K$ and therefore $F$ is concerned, all that the ghosts have done is to shift $\phi_{1}$ by $\eta_{1}+\eta_{2}$. As a result, eqs.(25) through (28) still 
continue to be valid, with the proviso that $\phi_{1}$ be replaced by the shifted $\phi_{1}$. These shifts do not change anything done in this paper, since we have never made any use of the actual value of $\phi_{1}$, and that is the reason why we have suppressed the ghost sector in the main body of the paper. We expect them to become important in the next non-leading order in $D$.

Although we do not need it, for the sake of completeness, we will carry out the functional integrations over $b, c, \bar{b}$ and $\bar{c}$ in $S_{g}$ (eq.(62)). The resulting $\operatorname{Tr} \ln$ is very simple:

$$
\operatorname{Tr} \ln _{g}=p^{+}\left(\tau_{f}-\tau_{i}\right) \frac{C}{16} \kappa_{1}^{2} \kappa_{2}^{2},
$$

where $C$ is a cutoff dependent constant. The reason for this simple answer is that the term $b^{\prime} \cdot c^{\prime}$ in (62), the only term that "propagates" the ghosts, does not contribute to the determinant. This is an example of the non-dynamical, or equivalently, non-propagating ghosts discussed in [9]. In the absence of propagation, the determinant, or the $T r \ln$ has purely algebraic dependence on the $\kappa$ 's. In any case, we see that the ghost contribution to the action is relatively trivial.

\section{References}

[1] S. S. Gubser, I. R. Klebanov and A. M. Polyakov, Nucl. Phys. B 636 (2002) 99 [arXiv:hep-th/0204051].

[2] J. M. Maldacena, Adv. Theor. Math. Phys. 2 (1998) 231-252, hepth/9711200.

[3] O.Aharony, S.S.Gubser, J.Maldacena, H.Ooguri and Y.Oz, Phys. Rept.323 (2000) 183 [arXiv: hep-th/9905111].

[4] K. Bardakci and C. B. Thorn, Nucl. Phys. B 626 (2002) 287 [arXiv:hepth/0110301]. 
[5] H. B. Nielsen and P. Olesen, Phys. Lett. 32B (1970) 203; B. Sakita and M. A. Virasoro, Phys. Rev. Lett. 24 (1970) 1146.

[6] G. 't Hooft, Nucl. Phys. B72 (1974) 461.

[7] C. B. Thorn, Nucl. Phys. B 637 (2002) 272 [arXiv:hep-th/0203167].

[8] S. Gudmundsson, C. B. Thorn, and T. A. Tran, arXiv:hep-th/0209102.

[9] K. Bardakci and C. B. Thorn, arXiv:hep-th/0206205. 